\title{
Optimization of Face Recognition Algorithms for Smartphone Environment
}

\author{
Kanghun Jeong, Dongil Han and Hyeonjoon Moon \\ School of Computer Science and Engineering, Sejong University, Seoul, Korea \\ E-mail: hmoon@sejong.ac.kr
}

\begin{abstract}
The face recognition algorithm has been investigated as a robust solution for biometric authentication. It is used in a wide range of applications such as surveillance, access control, airport immigration system and application of user recognition in a smartphone, etc. (The security of face recognition system can be trusted, because they were used unique biometric data) Face recognition system tends to give a higher degree of recognition performance only when image acquisition process was restricted environment. In this paper, we explore face detection and face recognition algorithms for smartphone environment. The basic requirements include appropriate data format and collection of feature data to achieve efficiency of algorithm. Our experiment presents a face detection and recognition algorithm for smartphone environment. The essential part for proposed system includes; (1) integer representation from floating point calculation on desktop computer and smartphone, (2) optimization of memory management scheme and (3) efficient face detection performance on complex background scene by Modified Census Transform.
\end{abstract}

Keywords: smartphone environment, face recognition, face detection, algorithm optimization. Integer representation, modified census transform

\section{Introduction}

In recent years, face detection and recognition technology has been developed rapidly. Face recognition is a task that humans perform routinely and effortlessly in our daily lives. Wide availability of powerful and low-cost desktop and embedded computing systems has created an enormous interest in automatic processing of digital images in a variety of applications, including biometric authentication, human computer interaction, access control, and user recognition application in smartphone. Research, development and performance enhancement in face recognition follows naturally.

Face recognition has several advantages over other biometrics modalities. Therefore, it is necessary to optimize the face recognition system by maintaining recognition accuracy while decreasing computational complexity in smartphone related application. However, most of the smartphones are hard to satisfy such factors because of limited configuration of memory and CPU power. The major factors of the face recognition system are dimension of the features and the processing efficiency. Generally, processing time is governed by geometric progression which is the dimensionality of feature data in the case of mobile-based devices. It is essential to optimize data structure while maintaining a reasonable recognition performance.

In this paper, we explore face detection and recognition algorithm for smartphone environment. Experiments include normalization of a face database to increase recognition 
performance through various pre-processing methods. We propose a novel algorithm for automatic face detection and post-processing method to further improve the face recognition performance for smartphone environment.

\section{Sejong Face Database (SJDB)}

Generally, face recognition system requires face image data that used for learning and features are normalized and registered for face recognition process. These face image data are collected for some time intervals in various conditions [1]. We have collected 3 set of face database for this experiment called "Sejong face database". A set of images of each person are used for learning and other sets are used for face recognition system. A session contains three images for each person (Figure 1).

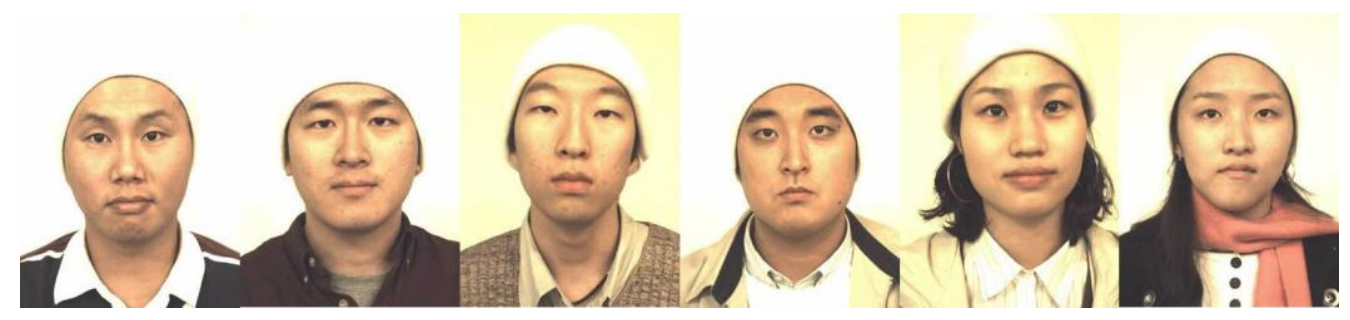

Figure 1. Sejong Face Image Database (SJDB)

The images of each person are collected one or two images a day in the same physical setup such as pose and lightning called 'session'. The session of $\mathrm{N}$ different people are collected without distinction of sex. In this way, we collected 100 different sessions about 70 male and 30 female. Each session contains three frontal images which were collected with similar illumination condition (170 220 lux) [2], [3].

\section{Face detection and face recognition}

For any face recognition system noise-data is a primary factor of degradation. Therefore, the process for remove noise is essential to improve recognition accuracy [3], [4]. This process is called pre-processing and it begins with geometric transformation (rotation, translation, and rescaling). The correction of the geometric transformation uses the intermediate point of the eyes and mouth. We remove the unnecessary data of the outer face region using an elliptical mask. The pre-processed image is properly modified with several feature point (eyes, nose, mouth) to have equal dimension regardless of personal face size [5] (Figure 2). The face images for this experiment has horizontal versus vertical ratio of 3:4. As a result, face images are modified to 1200 by 1600,120 by 160 , and 30 by 40 size.

Table 1. Pre-Process Time For One Face Data (Not Integerized)

\begin{tabular}{|c|c|c|c|}
\hline Image size & 1200 by 1600 & 120 by 160 & 30 by 40 \\
\hline $\begin{array}{c}\text { Processing time (ms) on } \\
\text { desktop computer }\end{array}$ & 1639.75 & 449.02 & 414.24 \\
\hline $\begin{array}{c}\text { Processing time (ms) on } \\
\text { smartphone }\end{array}$ & 4475.24 & 1064.88 & 849.58 \\
\hline
\end{tabular}




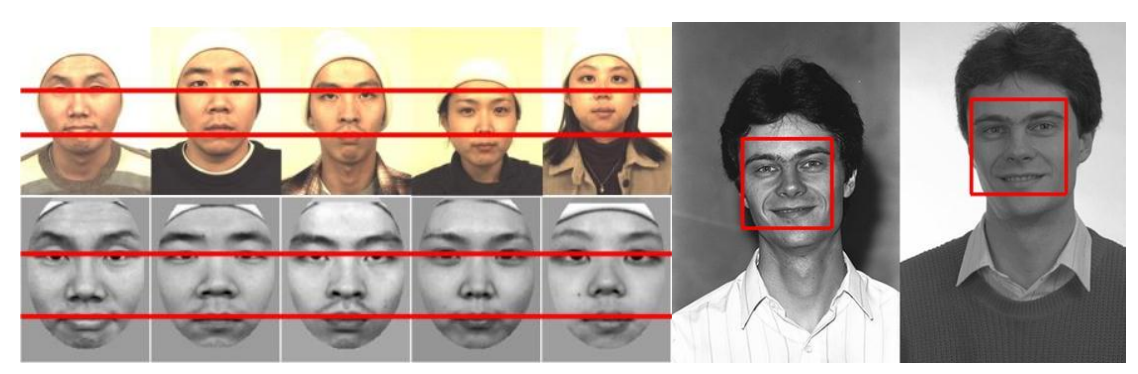

\section{Figure 2. Preprocessing Criteria, Processing and Modified Census Transform Results}

The pre-processing time is shown in Table 1 which was performed based on Intel Core ${ }^{\mathrm{TM}} 2$ i5 computer with $2.6 \mathrm{GHz}$ and 4GByte configuration and Samsung Galaxy SII smartphone environment. In this paper the pre-processing was performed with fully automatic setup with modified census transform (MCT) [6] and Adaboost based algorithm [7]. The image with size of 120 by 160 does the best performance reference of Table 1 . In this experiment, we have applied series of algorithms including histogram equalization and filtering. As for the image, noise data was removed and the feature data is emphasized. In addition, intensity information of the image was redistributed through histogram equalization. The structure of our system is shown in Figure 3.

We have used principal component analysis (PCA) [2] and linear discriminant analysis (LDA) for feature extraction [8]. As for the feature data, the change of recognition rate is presented with different cutoff ratio (percentage of feature vectors used) as shown in Table 2. We set the cutoff ratio at $80 \%$ and designed our face recognition system which we considered as most appropriate performance. We have measured the distance between feature vectors based on L2 norm. The choice of the automatic face detector was based on Adaboost algorithm which was trained using FERET, XM2VTS, CMU PIE and Sejong face database. The size of learning data is using face ( 22 by 22 ), eyes ( 10 by 6 ) and mouth ( 20 by 9 ) pixels. The 3,513 positive data and 10,777 negative pieces of image data are collected and used. As a result, we succeeded in the face detection from near frontal face images ranging from $0 \sim 15$ degrees.

\section{Table 2. A Face Recognition Result by the Change of Image Size and Cutoff} Ratio in Smartphone Environment

\begin{tabular}{|c|c|c|c|}
\hline Image size & 1200 by 1600 & 120 by 160 & 30 by 40 \\
\hline $100 \%$ & $96 \%$ & $98 \%$ & $97 \%$ \\
\hline $80 \%$ & $97 \%$ & $98 \%$ & $96 \%$ \\
\hline $70 \%$ & $94 \%$ & $95 \%$ & $91 \%$ \\
\hline
\end{tabular}

For learning the face detection data using Adaboost algorithm, negative (wrong) data is equally important as positive (correct) data. After appropriate training, the face detector can locate feature points (the center of each eyes and the center of the mouth) from a various rotated angle and front face image. After pre-processing of face image, face recognition takes about $7 \mathrm{fps}$ (frame per second) which is reasonable for real-time processing. The total processing time requires $1.1 \mathrm{fps}$ (frame per second) including the face detection. Face detection and face recognition process show rate of $87 \%$ detection and $95 \%$ recognition percentage each. 


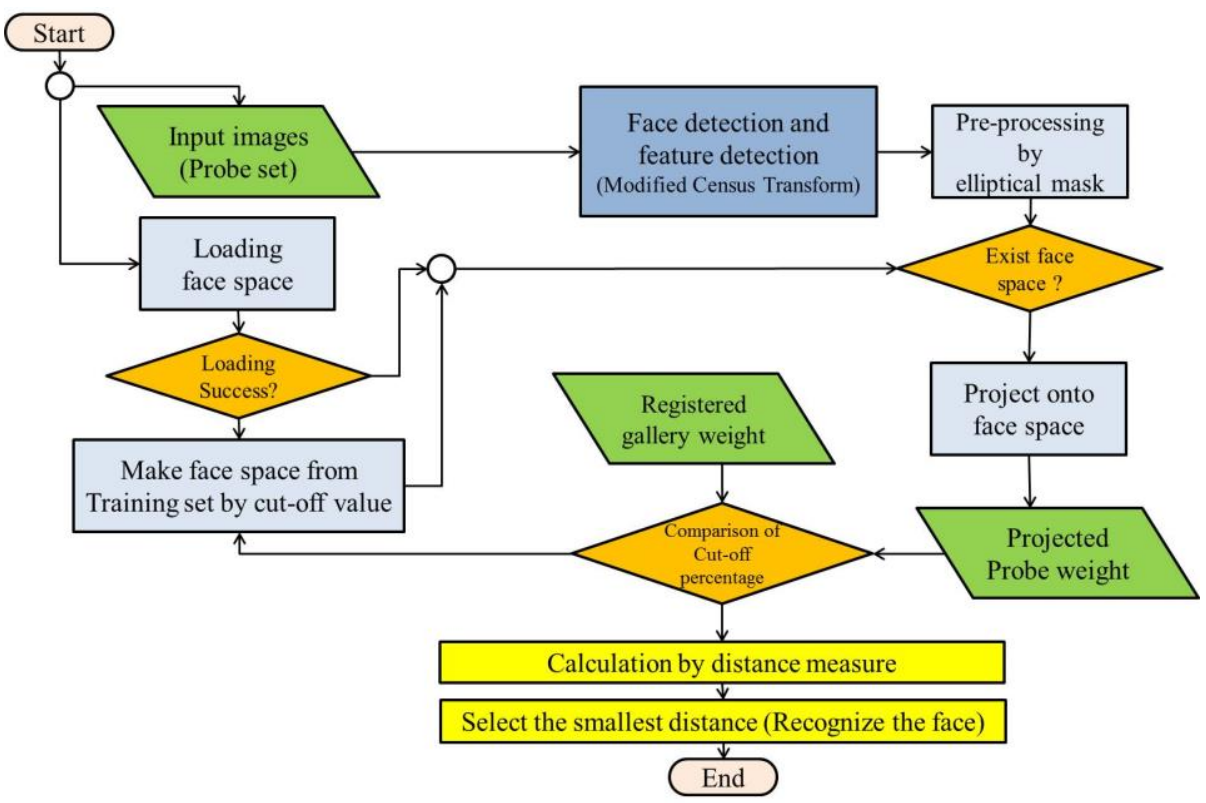

Figure 3. Structure of Proposed System

\section{Performance Profiling}

We have implemented four major face recognition functions to performed general smartphone environment. Biometric function is composed of face detection, face recognition and face evaluation module designed based on Google Android platform. Generally, face recognition algorithms are expressed by normalized numerical value. Such real number based algorithms are used for graphical algorithms such as image processing, 3D rendering, etc. There exists noticeable performance drops between floating and integer number based calculation. Moreover, this difference appears greatly on smartphone environment has weak floating point unit (FPU) compared to desktop computer.

Since face recognition algorithm use floating point based calculation algorithm, performance degradation can be significant. In order to reduce these differences, integer number type was used with fixed point method which is a numerical analysis method to store fraction's part of the decimal and the integer number separately. Fixed-point method was used for face recognition algorithm to produce faster than existing algorithm with floating algorithm. We designed fixed decimal point algorithm is designed to perform faster than floating point algorithm. There is proved measurement of a runtime through simple $\mathrm{C}$ program. Integral fragmentary performance can be verified through profiling. In practice, performance can be evaluated through integral conversion which uses fixed point algorithm. Face image data was used for performance evaluation with 120 by 160 pixels. Evaluation unit compare each module during the process. Change of total arithmetic velocity depends on integral transform considerably. This fact is that because additional arithmetic is contained through integral transform, and performance improvement is guaranteed if it can be applied on smartphone environment as shown in Table 3 and Table 4 (smaller numbers faster). 
Table 3. 7,000,000 Times of Profiling Repetition Results on (a) Intel ${ }^{\circledR}$ Core ${ }^{\text {TM } 2}$ i5 2.6GHz and (b) Intel ® Pentium - 233

\begin{tabular}{|l|c|c|c|c|c|c|}
\hline & \multicolumn{2}{|c|}{ Integer number } & \multicolumn{2}{c|}{ Floating number } & \multicolumn{2}{c|}{ Double number } \\
\hline Test environment & (a) & (b) & (a) & (b) & (a) & (b) \\
\hline Addition & 62.1 & 274.722 & 89.1 & 270.63 & 255.1 & 280.31 \\
\hline Subtraction & 78.2 & 267.69 & 87.6 & 269.54 & 277.6 & 282.80 \\
\hline Multiplication & 113.3 & 274.09 & 447.2 & 3702.76 & 997.1 & 3702.03 \\
\hline Division & 311.2 & 1379.03 & 490.5 & 4053.54 & 817.7 & 4022.88 \\
\hline
\end{tabular}

Table 4. Face Recognition Time in Desktop Computer and Smartphone Environment. 1200 by 1600 was not Experiment, because Face Space File Size is too Large To Load into Memory

\begin{tabular}{|c|c|c|c|}
\hline Image size & $1200 \times 1600$ & $120 \times 160$ & $30 \times 40$ \\
\hline $\begin{array}{c}\text { Face recognition } \\
\text { time (msec) }\end{array}$ & $649.06(-)$ & $\begin{array}{c}17.79(38.03 \mathrm{~ms} \\
\text { in smartphone })\end{array}$ & $\begin{array}{c}0.54 \\
(0.87 \mathrm{~ms} \text { in } \\
\text { smartphone })\end{array}$ \\
\hline $\begin{array}{c}\text { Face space file } \\
\text { size }\end{array}$ & 1.551 GByte & $15.001 \mathrm{MByte}$ & $0.938 \mathrm{MByte}$ \\
\hline
\end{tabular}

\section{Conclusion}

The face recognition system is essential in multimodal biometrics system since it provides the only non-contact biometric features and user friendly information which can be processed by both human and computer. This is very important feature in case of communication problem caused by network failure which prohibits database access for smartphone related applications. Recently, face detection and recognition technology can be built into an embedded system for mobile devices. However, this system should respond to numerous conditions including various pose and lighting which is challenging for face recognition system. Generally, a face recognition system contains two major functions which is face detection and face recognition. We produce several face detection and recognition algorithms in smartphone environment for various biometric applications.

We present performance evaluation for integral transformed calculation which is optimized for smartphone environment. We have trained face detection using modified census transform (MCT). Face recognition modules are based on principal components analysis (PCA) and linear discriminant analysis (LDA). Proposed face detection and recognition methods are integral transformed which can be calculated faster, and optimized for smartphone environment. Our experimental result shows that we can minimize the run-time by decreasing computational complexity while maintaining reasonable accuracy which is applicable for smartphone environment.

\section{Acknowledgements}

This research was supported by Basic Science Research Program through the National Research Foundation of Korea (NRF) funded by the Ministry of Education, Science and Technology (2011-0026048). Hyeonjoon Moon (hmoon@sejong.ac.kr) is the corresponding author. 


\section{Reference}

[1] T. Papatheodorou and D. Rueckert, "Evaluation of 3D Face Recognition Using Registration and PCA", AVBPA 2005, LNCS 3546, (2005), pp. 997-1009.

[2] M. Turk and A. Pentland, "Eigenfaces for recognition", J. Cognitive Neuroscience, vol. 3, (1991), pp. 71-86.

[3] H. Moon and P. Phillips, "Computational and Performance Aspects of PCA-Based Face-Recognition Algorithms", Perception, vol. 30, (2001), pp. 303-321.

[4] T. Sim, S. Baker and M. Bsat, "The CMU Pose, Illumination, and Expression Database", IEEE Transactions on Pattern Analysis and Machine Intelligence, vol. 25, no. 12, (2003), pp. 1615-1618.

[5] P. Phillips, H. Moon and P. Rauss, "The FERET Evaluation Methodology for Face Recognition Algorithms", IEEE Transactions on Pattern Analysis and Machine Intelligence, vol. 22, no. 10, (2000), pp. 1090-1104.

[6] B. Froba and A. Ernst, "Face detection with the modified census transform", Sixth IEEE International Conference on Automatic Face and Gesture Recognition, Proceedings, (2004) May, pp. 91-96.

[7] P. Viola and M. Jones, "Rapid object detection using a boosted cascade of simple features, in Computer Vision and Pattern Recognition", (2001), pp. 511-518.

[8] W. S. Yambor, "Analysis of PCA-based and Fisher Discriminant-based Image Recognition Algorithms", technical report, CSU, vol. 6, (2000).

\section{Authors}

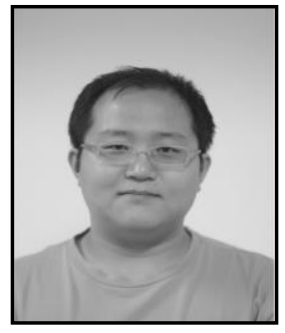

Kanghun Jeong received a B.S. degree from Sejong University, Seoul, in 2006, an M.S. in 2008, and is currently continuing a Ph.D. degree in Computer Science and Engineering from Sejong University. His research interests include computer vision, pattern recognition, multimodal biometrics, information security and face detection/recognition based on a smartphone environment.

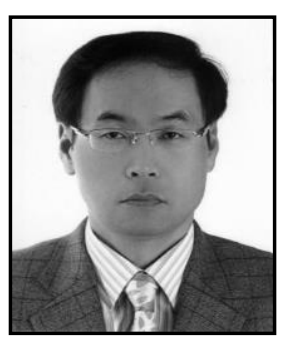

Dongil Han received the B.S. degree in electronics and computer engineering from Korea University, Seoul in 1988 and the M.S. and $\mathrm{Ph} . \mathrm{D}$. degree in electrical and electronics engineering from Korea Advanced Institute of Science and Technology, Seoul, Korea in 1990 and 1995, respectively. From 1995 to 2003, he was a chief research engineer at digital TV R\&D Laboratories in LG Electronics Inc., Seoul, Korea. He is currently a professor in the Department of Computer Engineering at Sejong University in Seoul, Korea. His research interests include image processing, display quality enhancement for digital TV, system on chip, and robot vision.

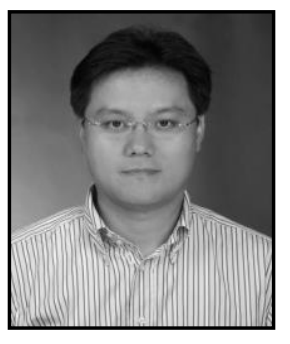

Hyeonjoon Moon received a B.S. degree in Electronics and Computer Engineering from Korea University, Seoul, in 1990, an M.S., and Ph.D. degrees in Electrical and Computer Engineering from the State University of New York at Buffalo, in 1992 and 1999, respectively. From 1993 to 1994, he was a systems engineer at Samsung Data Systems in Seoul, Korea. From 1996 to 1999, he was a research associate at the US Army Research Laboratory in Adelphi, Maryland. From 1999 to 2002, he was a senior research scientist at Viisage Technology in Littleton, Massachusetts. Currently, he is a professor of Computer Science and Engineering at Sejong University in Seoul, Korea. His research interests include computer vision, pattern recognition and biometrics. 